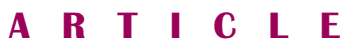

\section{Building a Computer Program to Support Children, Parents, and Distraction During Healthcare Procedures}

\author{
KIRSTEN HANRAHAN, ARNP, DNP \\ ANN MARIE McCARTHY, PhD, RN \\ CHARMAINE KLEIBER, PhD, RN \\ KAAN ATAMAN, PhD \\ W. NICK STREET, PhD \\ M. BRIDGET ZIMMERMAN, PhD \\ ANNE L. ERSIG, PhD, RN
}

Researchers often rely on multiple measures to predict complex human behaviors. Children's distress responses to common healthcare procedures, such as immunizations and needle sticks, may be affected by multiple factors such as child temperament, state and trait anxiety, coping style, parents' anxiety and parenting style, and other variables. ${ }^{1}$ Adding further to the complexity, it may require dozens

Author Affiliations: College of Nursing, University of lowa, Nursing Research and Evidence-Based Practice, University of lowa Hospitals and Clinics, lowa City (Dr Hanrahan); College of Nursing, University of lowa (Drs McCarthy and Kleiber); Argyros School of Business and Economics, Chapman University, Orange, California (Dr Ataman); and Tippie College of Business (Dr Street), Biostatistics Consulting Center, Department of Biostatistics (Dr Zimmerman), and College of Nursing (Dr Ersig), University of lowa.

This study was funded by R01 grant NR05269-01A2 from the National Institute for Nursing Research.

The authors have disclosed that they have no significant relationship with, or financial interest in, any commercial companies pertaining to this article.

Corresponding author: Kirsten Hanrahan, ARNP, DNP, College of Nursing, University of lowa, Nursing Research and Evidence-Based Practice, University of lowa Hospitals and Clinics, 200 Hawkins Dr, T 140 CNO, lowa City, IA 52242 (kirsten-hanrahan@uiowa.edu).

Supplemental digital content is available for this article. Direct URL citations appear in the printed text and are provided in the HTML and PDF versions of this article on the journal's Web site (www.cinjournal.com).

DOI: 10.1097/NXN.0b013e31825e211a
This secondary data analysis used data mining methods to develop predictive models of child risk for distress during a healthcare procedure. Data used came from a study that predicted factors associated with children's responses to an intravenous catheter insertion while parents provided distraction coaching. From the 255 items used in the primary study, 44 predictive items were identified through automatic feature selection and used to build support vector machine regression models. Models were validated using multiple cross-validation tests and by comparing variables identified as explanatory in the traditional versus support vector machine regression. Rule-based approaches were applied to the model outputs to identify overall risk for distress. A decision tree was then applied to evidencebased instructions for tailoring distraction to characteristics and preferences of the parent and child. The resulting decision support computer application, titled Children, Parents and Distraction, is being used in research. Future use will support practitioners in deciding the level and type of distraction intervention needed by a child undergoing a healthcare procedure.

\section{KEYWORDS}

Children • Data mining • Decision support • Distress • Methods $\cdot$ Parent $\cdot$ Procedure

of questions to quantify complicated factors such as temperament or coping style, and it is not practical for busy clinicians to use extensive research measures to predict a child's distress response to a procedure. Clinicians need brief, reliable screening tools that can be used to predict a response so that interventions can be tailored to the individual child in the clinical setting.

Pain and distress related to healthcare procedures are a significant problem for most children. Children who experience inadequate pain control during medical procedures can suffer immediate and long-term negative sequelae. ${ }^{2,3}$ Research has shown that the immediate effects of minimizing child pain and distress during a procedure include a decrease in child and parent emotional stress, a higher rate of successful intravenous (IV) catheter insertions on the first attempt,${ }^{4}$ and decreased child 
physiological stress response. ${ }^{5,6}$ Distraction, which is changing the individual's focus of attention, is an evidence-based intervention that can be used to decrease child distress during painful medical procedures. ${ }^{7,8}$ Parents can be instructed to coach their child in distraction using nonprocedural talk, books, bubbles, and other novelty toys to capture the child's attention. Children differ in the level of distress experienced and in their response to interventions designed to decrease their distress. To ensure that appropriate interventions are initiated, clinicians need practical decision support systems that (1) predict a child's response to a painful procedure when parents provide distraction coaching, (2) recommend the level of support and resources needed to facilitate distraction, and (3) provide evidence-based tailored instructions for the parent and child.

The purpose of this article was to describe the development of a decision support system, Children, Parents, and Distraction $(\mathrm{CPaD})$. This computer-based program predicts the level of a child's distress when parent distraction is provided during a healthcare procedure and identifies tailored instructions for parents. The first step in developing $\mathrm{CPaD}$ was the secondary data analysis of an existing data set using data mining methodology to develop predictive models that identify risk for distress in children during a healthcare procedure and parent ability to provide distraction coaching to their child. Data mining is a method designed to search for consistent patterns and relationships among items in a large data set. These patterns are put into a "model" that can be validated and refined by other test cases and then applied to predict responses for new cases. ${ }^{9}$ Once the predictive models were identified, the second step in building the $\mathrm{CPaD}$ decision support was to develop a rule-based, clinically derived approach to assign children to groups stratified by overall risk for distress in the clinical setting. The final step was to apply a decision tree for evidence-based instructions for tailoring distraction to characteristics and preferences of the parent and child. These three components were combined and programmed for the computerbased decision support application, $\mathrm{CPaD}$. Predictive validity was evaluated in a clinically based research study.

\section{$\square$ STEP 1: DATA MINING AND PREDICTIVE MODEL BUILDING}

In this secondary data mining analysis, data were used from a randomized clinical trial carried out to identify child, parent, and procedural factors that explain children's responses to parent-provided distraction coaching during a healthcare procedure. ${ }^{10}$ In the study, parents were randomly assigned to either an intervention group, in which they were provided with distraction training
(DT), or to a control group, in which they had no training on using distraction (no DT). Data were obtained on variables identified in the literature as potentially explaining a child's response to parent-provided distraction coaching. ${ }^{1}$ Outcome measures of the child's distress response to an IV insertion included (1) behavioral distress using the Observational Scale of Behavioral Distress-Revised (OSBD-R), ${ }^{11}$ (2) physiological response using salivary cortisol levels, ${ }^{12,13}$ (3) parent perception of the child's distress using one item from the Perception of Procedures Questionnaire, ${ }^{14}$ and (4) child report of pain using the Oucher. ${ }^{15,16}$ In addition, the level of distraction provided by a parent was measured by a behavioral observation scale, the Distraction Coaching Index (DCI). ${ }^{17}$ Figure 1 and other reports ${ }^{10,18}$ describe the study methods and results from the primary analyses. The institutional review boards at each data collection site approved this study. All data were deidentified for the secondary analysis, and therefore no further review for the protection of human subjects was indicated.

In order to develop predictive models, knowledge discovery in databases (KDD) or data mining techniques ${ }^{19,20}$ were used. Table 1 includes a list of data mining terms (italicized in this article text) and definitions. Predictive data mining uses specific cases with known outcomes to construct models that use independent variables (such as demographics and survey responses) to predict outcomes for future cases. The predictive models were built using support vector machine (SVM) regression as implemented in the Weka (University of Waikato, Hamilton, New Zealand) data mining package. ${ }^{21,22}$ Generalization, which is the performance of the model on unseen data, was further improved by an automatic feature selection method. Using this method, individual items are automatically selected from the data to build the best models for predicting outcomes. Together, the set of predictive models maps child and parent characteristics to three predicted outcomes.

\section{System Design}

Data from the study were used in a secondary analysis to test variables for predicting outcomes with new parents and children. Four models using a subset of the parent, child, and procedural variables predict three outcomes: coachability (DCI), behavioral distress (OSBD-R), and physiological distress (cortisol responsivity). The ability of the parent to provide distraction (coachability) was predicted from two submodels that used data from the no DT and DT groups to predict DCI scores. Two other outcomes, the Oucher and parent perception of child distress, did not increase the overall predictive ability and therefore were not included. The predictive models (Figure 2) are as follows: 


\section{Children's Responses to Medical Procedures When Parent Distraction is Provided}

Purpose: Identify parent, child and procedural variables that explain children's pain and distress responses to an IV insertion when parent distraction (using books, toys, non-procedural talk to move child's focus away from noxious stimulus) is provided.

Design: A multi-site Randomized Clinical Trial (RCT) comparing an intervention group in which parents received a basic distraction intervention (including a 7 minute training video, a brief coaching session, and helping and choosing distraction items for the child) to a control group receiving routine care. Both groups had a basket of distraction items available during the procedure.

Sample: $\quad$ A convenience sample of children, 4 to 10 years of age, English speaking and undergoing an intravenous (IV) needle insertion for a diagnostic medical procedure and their parents

\section{INSTRUMENTS}

Explanatory Variables and Measures

Child

Demographics Pre-Procedure Questionnaire (age, gender, ethnicity)

Illness severity Severity of Illness Scale

State and trait anxiety Thermometer Visual

Analogue Scale

Anxiety/attention/behavior Pediatric Behavior Scale

Temperament Dimensions of Temperament

Coping style Child Behavioral Style Scale

Genetics Genotyping of candidate genes for pain

response

\section{Parent}

Demographics Pre-Procedure Questionnaire (age, gender, ethnicity)

State and trait anxiety State Trait Anxiety Inventory

Parenting style Parenting Dimensions Inventory

\section{Procedural}

Difficulty of IV insertion Degree of difficulty question- 4 point scale

Use of topical anesthetic Dwell time from application to removal compared to product recommended dwell time.

Child behavioral distress Observation Scale of

Parent perception of child distress Revised Perception of Procedures Questionnaire (PPQ)

Child stress Salivary Cortisol levels

Child self-report of pain Oucher Scale

Parent distraction coaching Distraction Coaching Index

DATA ANALYSIS

Intervention efficacy Contingency table analysis, rank sum test, \& logistic regression.

Explanatory models Logistic regression

\section{RESULTS}

Sample A total of 542 children, $262(48 \%)$ girls, $280(52 \%)$ boys, with a mean age of 6.95 years (SD 1.90) participated. Parent coaches were primarily white $(84 \%)$, mothers $(88 \%)$, with some college education $(71 \%)[7]$.

Findings Children of parents who provided high quality distraction coaching demonstrated less behavioral distress, less cortisol response, and lower parent report of child distress compared to children whose parents provided low quality distraction [1].

Child, parent and procedural variables that potentially explain children's responses to parent distraction coaching during a medical procedure were identified [2]

FIGURE 1. Synopsis of study and primary analysis.

\section{Distraction Coaching Index Predictor: No Distraction Training}

The DCI Predictor (model 1) for parents with no DT was developed using cases from the study's no DT group. In this model, parent variables are used to predict what a parent's DCI scores would be if no training in how to provide distraction coaching was given. The intent of this predictor is to generate a baseline expected score for a parent's ability to provide distraction.

\section{Distraction Coaching Index Predictor: Distraction Training}

The DCI Predictor for parents receiving DT (model 2) uses data from the study DT group to predict a parent's ability to be a distraction coach (DCI scores) when training is provided. For a given set of parental characteristics, the difference between the two DCI Predictor components can produce an estimate of "coachability" or the predicted improvement of the parent's ability to provide distraction coaching to the child if training is provided. The DCI differential or coachability is the first risk output. High coachability predicts that the parent will improve in distraction ability if training in distraction is given, while low coachability predicts that training is not likely to change the parent's ability to provide distraction.

\section{Behavioral Distress Predictor}

The OSBD-R Predictor (model 3) predicts child behavioral distress in future cases. Predicted OSBD-R is obtained using parent and child variables as inputs and the predicted DCI score from the study DT group. The 
T a b I e 1

Data Mining Terms and Definitions

Term

Definition

Automatic feature selection method

Evaluates the worth of a subset of items by considering the individual predictive ability of each item along with the degree of redundancy between them; performs a combination of forward or backward search through the space of item subsets

Cross-validation

A repeated hold-out evaluation method in which models are built using $90 \%$ of the cases and tested on the remaining, held-out, 10\%; this is repeated 10 times, such that every subject is used once as a test case

Data mining techniques

The process of extracting patterns from large data sets to determine relationships between variables

The Gaussian kernel parameter that controls the amount up to which deviations are tolerated in the regression

Gaussian kernels

KDD

Statistical functions for normal curves

Process of discovering useful knowledge from data; includes methods for data mining, but also encompasses data preparation, data selection, data cleaning, applying previous knowledge, and interpreting data to ensure that useful knowledge comes from the data

MSE

A standard performance measure for regression problems and was used to compare the performance of our predictors with linear least-squares regression models

SVM regression

Constructs highly nonlinear regression models and tolerates small fitting errors to improve the generalization of the resulting predictor on unseen data

Weka

Software package for data mining (University of Waikato)

$\gamma$

The Gaussian kernel parameter that controls the complexity of the model; greater values lead to models that more closely resemble a linear fit to the data, while smaller values capture nonlinear relationships between the predictive variables and the outcome

second output for the model is a predicted OSBD-R score or risk for child behavioral distress.

\section{Physiological Distress Predictor}

The Cortisol Predictor (model 4) predicts child physiological response for future cases. The child's cortisol level is predicted using the parent and child variables, predicted DCI, and one procedural variable, use of topical analgesic. This leads to the third output, predicted cortisol response or risk for physiological distress.

\section{Model Construction}

The process for constructing each model follows the same basic form. The first step was to apply an automatic

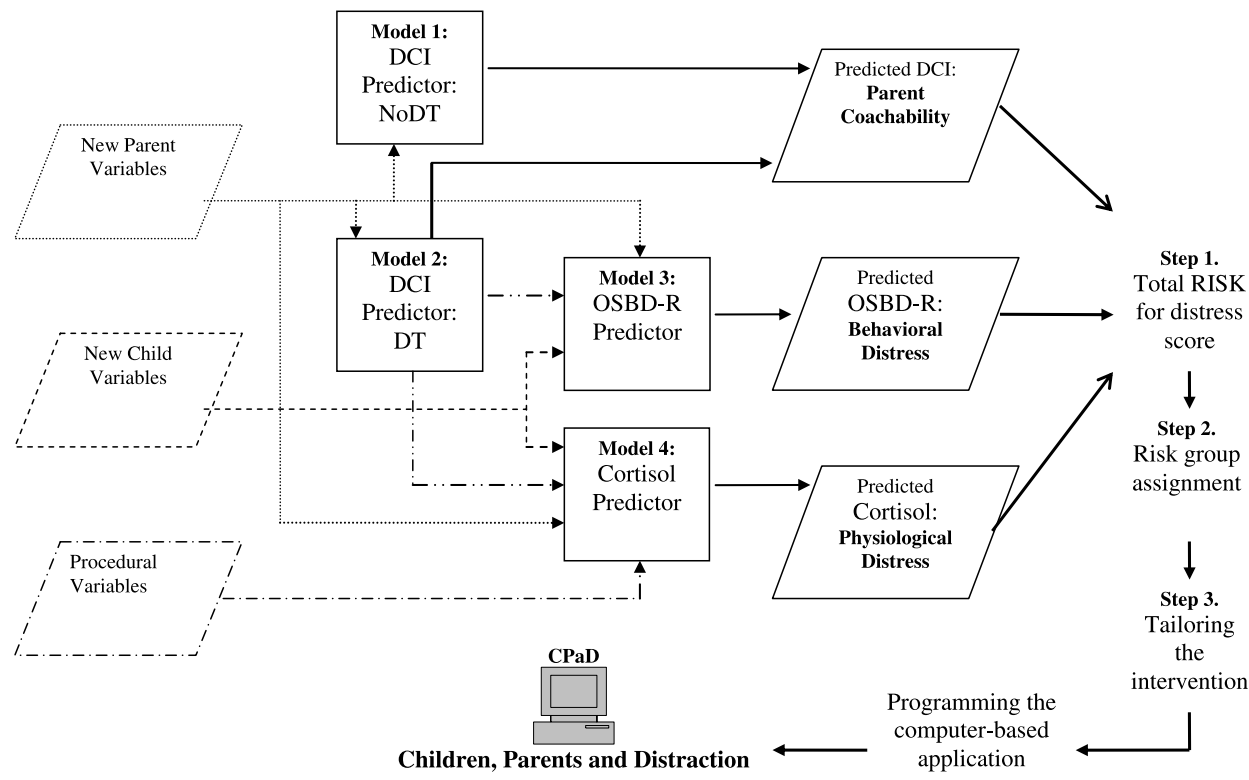

Note: DT = distraction training, DCI = Distraction Coaching Index, OSBD = Observed Scale of Behavioral Distress-Revised

FIGURE 2. Development of the predictive models and steps for building CPaD decision support program. 
T a b I e 2

Examples of Items Included in the Predictive Models of Distress

Model

Example Items ${ }^{a}$

(1) DCl Predictor, no intervention

Have you ever used distraction to help your child cope with a painful event?

I am tense.

Our family is organized and "together."

Father's ethnicity

(2) DCI Predictor, basic intervention

Once I decide how to deal with misbehavior of my child, I follow through on it. Do you think you will be effective in using distraction during today's IV insertion? Mother's education

(3) OSBD predictor

Has your child ever had an IV?

How do you expect your child to behave during today's IV insertion?

How does your child typically cope with pain?

Does your child never seem to stop moving?

I often notice how my body feels. ${ }^{\text {b }}$

(4) Cortisol predictor

When thinking about the IV insertion today, how actively involved do you like to be in helping your child?

Is your child generally fearful, anxious, or worried?

Expected dwell time for topical anesthetic

aparent report items except where noted.

${ }^{\mathrm{b}}$ Child report items.

feature selection method. ${ }^{21}$ Each model was constructed using SVM regression with Gaussian kernels. ${ }^{23}$ Data were collected on 255 items in the study, and automatic feature selection identified 44 items to be used as inputs in one or more of the four predictive models (Table 2). For optimization of the SVM regression performance, we utilized two parameters, $\gamma$ and $\varepsilon$. Optimal parameters for each model were chosen by a greedy search of the parameter space (Table 3 ).

\section{Validation Process}

Each model was extensively evaluated using multiple crossvalidation tests. By averaging the results, we achieve an accurate and nearly unbiased estimate of model performance on unseen data. Mean square error (MSE) and SD of MSE on hold-out cases are shown in Table 4, estimated by averaging the 10 separate runs of crossvalidation. Performance on held-out cases was significantly better than linear regression in every case $(P<.01)$,

\section{T a b I e 3}

Optimal Model Parameters

\begin{tabular}{lll} 
Model & $\gamma$ & \multicolumn{1}{c}{$\boldsymbol{\varepsilon}$} \\
\hline Control DCl & 0.2 & 0.001 \\
Intervention DCl & 0.006 & 0.002 \\
OSBD & 0.04 & 0.0001 \\
Cortisol & 1 & 0.02 \\
\hline
\end{tabular}

$\gamma$ Is the Gaussian kernel parameter that controls the level of nonlinearity of the model; $\varepsilon$ is the parameter that controls the amount up to which deviations are tolerated in the regression. meaning predictive accuracy of the SVM regression models was superior to linear regression.

The model was also validated by comparing variables identified as explanatory in the traditional multiple regression (primary) analysis with variables identified through the vector SVM regression. Multiple regression analyses identified 30 items that explained the responses of the children in the primary study, while data mining identified 44 items for predicting child behavior in future cases. Item-by-item comparisons showed many of the variables identified as being statistically significant by the explanatory linear regression analyses for OSBD-R and cortisol were also identified by the feature subset selection method and included in the predictive models.

\section{STEP 2: RISK GROUP ASSIGNMENT}

The next step in developing the decision support system was to translate the data mining outputs into meaningful clinical information. A rule-based approach was applied to the three SVM regression outputs (ie, coachability,

\section{T a b I e 4}

Test MSE and (SD) for Predictive Models

\begin{tabular}{lcr} 
Data Set & $\begin{array}{c}\text { Linear Regression } \\
\text { Test MSE }\end{array}$ & $\begin{array}{c}\text { SVM Regression } \\
\text { Test MSE }\end{array}$ \\
\hline Control DCI & $8.330(0.224)$ & $7.446(0.124)$ \\
Intervention DCI & $12.059(0.314)$ & $10.278(0.056)$ \\
OSBD & $4.464(0.121)$ & $3.421(0.012)$ \\
Cortisol & $12.305(0.298)$ & $6.387(0.115)$ \\
\hline
\end{tabular}


behavioral distress, and physiological distress) in order to assign new individual parent-child dyads to groups based on risk of distress. Scores from each output were categorized into three approximately equal-size groups of high, medium, and low risk. For DCI differential, the high-risk group (ie, parents with low coachability scores) was defined by expected changes in the small range 0 to 10.15; the low-risk group had expected changes above 14 , and the medium-risk group consisted of those in between. The intervals for predicted OSBD-R were as follows: low risk, 0 to 1.075; medium risk, greater than 1.075 to 3.19; and high risk, greater than 3.19. Actual OSBD-R scores were from 0. Predicted cortisol ranges were as follows: low risk, 0 to 2.2; medium risk, greater than 2.2 to 3.83 ; and high risk, greater than 3.83 . Validity for the cutoffs was supported by a panel of clinical experts that reviewed videotapes of subjects at cutoff points.

Next, scores from the model's three outputs needed to be combined into an overall risk of distress score for the future parent-child dyads. Again, a rule-based system was applied to combine the risk assessments (high, medium, or low) of the three outcomes into an overall assessment of risk for distress for each possible combination of outputs. Rules for decision making may be based on empirical data, clinical judgment, or a need to manipulate group size. In this case, a combination of these strategies was used. A numerical value of 3,2, or 1 was assigned, respectively, to high, medium, and low risks for DCI and cortisol. Because behavioral distress was the primary indicator of total child distress in the study, OSBD-R was given greater weight and assigned values of 5 for high, 3 for medium, and 1 for low risk. Total risk for child distress was computed by summing the three outputs. Total scores were considered high risk for distress if they were greater than 8 , medium if 5 to 8 , and low if less than 5 . For example, a case with low predicted coachability (corresponding to the high-risk group, score $=3$ ), medium predicted OSBD-R (score $=3$ ), and high predicted cortisol level (score $=3$ ) would be placed in the high-risk group (total score $=9$ ). There are a total of 27 combinations of the three output models (DCI, OSBD-R, and cortisol) with the three possible distress levels (high, medium, low) for risk group assignment. When the rules were retrospectively applied to the study sample of 542 families, the distribution of children in the predicted high, medium, and low distress groups was $22 \%, 65 \%$, and $13 \%$, respectively. Levels of intervention to support distraction, based on the availability of local resources, could be then be allocated according to the individual child's risk for distress.

\section{STEP 3: TAILORING THE INTERVENTION}

The final step in developing the decision support system was to build the capacity to tailor evidence-based instructions, and intervention, based on characteristics and preferences of the parent and child. Some of the items used to assess risk could also be used to tailor the intervention. An evidence-based guideline for tailoring the distraction intervention was developed by the investigators; from this guideline, a decision tree was used to tailor teaching points to parent and child characteristics and preferences. For example, if a parent responds that the child prefers to use books for distraction and prefers to look away during the procedure, specific directions are included for how to use books to distract the child and shield the child's view of the procedure at the same time. Individualized outputs for each child are created from the decision tree based on answers to the questions.

\section{PROGRAMMING FOR THE COMPUTER-BASED APPLICATION}

These three components were then combined by the research team into a Web-based decision support system, $\mathrm{CPaD}$, available at any location where there is Internet access. The primary functions of the application are to (1) predict child risk for distress in response to healthcare procedure; (2) identify the level of support the parents need in order to provide distraction to the child, based on risk for distress; and (3) provide tailored educational material for the family. Additional functions to facilitate randomization and data management for research purposes were also included. The completed $\mathrm{CPaD}$ decision support system includes 44 predictive items plus 40 additional items to support tailoring and further research, for a total of 84 items. Computer programming and interfacing including screens with pull-down menus, foils, toggles, and graphics to prompt responses and guide the user were developed to provide a simple user-friendly interface for the clinical setting (Figure 3). Information is provided to the family both electronically and as an individualized handout that they can take into a treatment room and have available for future procedures. To access a prototype of the $\mathrm{CPaD}$, follow the link and instructions provided (See Document, Supplemental Digital Content 1, http://links.lww.com/CIN/A2).

\section{CLINICAL VALIDATION}

Initial validation to predict child distress in a clinical setting is supported by data from a recently completed follow-up study of child distress during an IV insertion. Of the 159 parents receiving DT (as described in the primary study), $\mathrm{CPaD}$ classified 45 children as low risk for distress and 114 children as high risk for distress. Analysis of the distress behaviors (OSBD-R scores) showed 


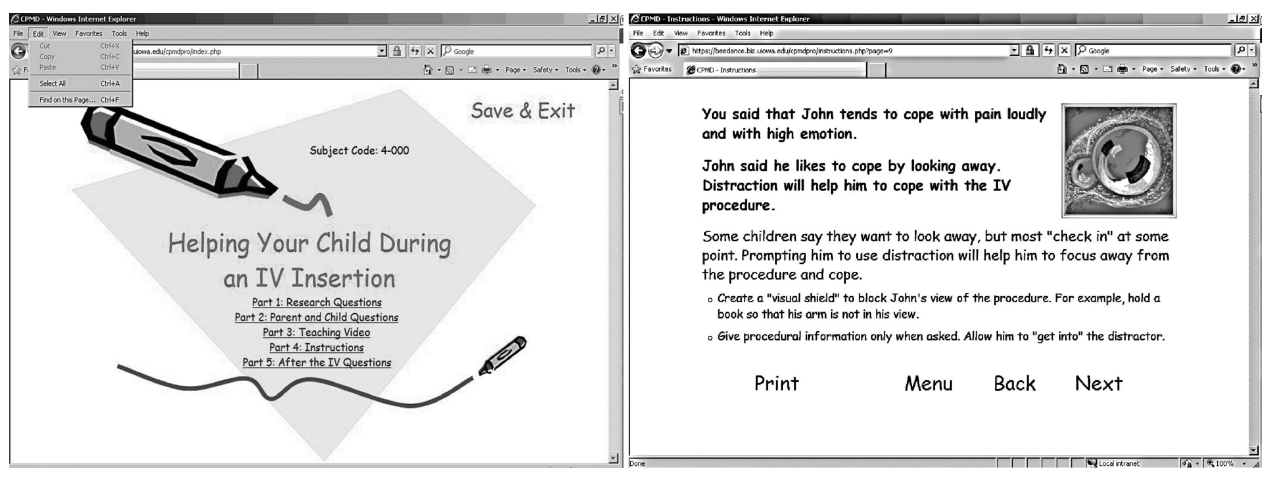

FIGURE 3. Screenshot of $\mathrm{CPaD}$ decision support program.

that $40 \%$ of children had an OSBD score that was within 1 of the predicted OSBD from $\mathrm{CPaD}$, with an additional $36 \%$ differing from predicted OSBD by no more than 1 to 3 points. There was a significant difference $(P=.006)$ between dyads predicted to be low versus high (ie, median OSBD-R scores of 0.67 versus 1.32), indicating that the $\mathrm{CPaD}$ was effective in identifying risk category. The follow-up study took place in an active pediatric outpatient clinic, where parents and children completed the questions on the Web site in approximately 10 minutes. Both clinic staff and families found $\mathrm{CPaD}$ engaging and useful. Parents and children report they prefer the computer application to traditional methods for giving and receiving information.

\section{DISCUSSION}

Many of the clinical problems confronting nurses have multiple etiologies or contributing factors. As illustrated in this article, child response to an invasive healthcare procedure and the effectiveness of parent distraction coaching to reduce child distress are influenced by numerous factors. These include child age, anxiety, temperament, and coping strategy; parent anxiety, expectations, distraction coaching behavior, and parenting style; and procedural difficulty and use of local anesthetics at the IV insertion site. Traditional regression procedures are of some utility for explaining factors accounting for responses, whereas data mining predicts responses for new cases. In order to develop a screening tool to identify those patients most in need of intervention, predictive modeling was needed. Data mining does not replace traditional statistical procedures but offers subtle pattern recognition that may not be intuitive. ${ }^{24}$ Using data mining techniques to systematically examining each of the 255 items in the original data set, we reduced the salient predictive items to just 44 .

The KDD process demonstrated significantly better predictive accuracy than the traditional statistical modeling approach. The flexibility of the SVM model allows highly nonlinear predictors, which can automatically incorporate interactions among the independent variables. This flexibility can be necessary when modeling a complex, poorly understood phenomenon such as reaction to pain. With properly chosen parameters, the SVM model achieves this flexibility while still generalizing well to new cases.

The accuracy and flexibility of the SVM method come at the expense of interpretability. The constructed models cannot be directly interpreted in the same sense that a linear regression model or a decision tree can be. Hence, a sensitivity analysis would be required to tease out the specific effects of the various independent variables. The approach is also affected by the number of training cases; in general, more cases lead to better predictions, and the relatively small number of cases in this study limits the predictive accuracy. Further, the addition of new cases requires occasional retraining of the models. Since families in the follow-up study all receive some level of distraction based on $\mathrm{CPaD}$, all future cases will have received an intervention of some sort, and the "DCI: No Intervention" model will receive no new training cases. Moreover, different levels of intervention explored in the follow-up study are not explicitly modeled. Thus, in order to stay current, the model will need to be retrained and the architecture adjusted.

Clinical judgment is necessary to turn the results of the data mining process into clinically useful decisions. Decision rules are based not only on empirical data, but also on the fit of data to risk groups. In this study, the different combinations of low, medium, and high risk for child behavioral and physiologic distress and parent coachability were weighted and assigned to overall risk for child distress. Expert clinicians on the research team decided that the child's predicted behavioral distress level should carry more weight in assigning overall risk than predicted parent coachability or child physiologic response. This decision was based on the team's years of clinical experience with children undergoing painful procedures. Team members observed how difficult it could be to change children's distress responses after a pattern of behavior is established over time. Children who have 
repeated bad experiences with healthcare procedures and who respond with high distress are more likely to continue with high behavioral distress. ${ }^{2,3}$ This observation was important for assigning risk for future distress.

Recently completed research used the $\mathrm{CPaD}$ computer application to assign dyads into risk groups in order to test three levels of distraction interventions. The Webbased application also serves as a database for research purposes, eliminating the need for data entry and double entry, thereby decreasing entry errors. Now that this research is completed, the models will be retrained. Further validation in other settings is needed.

In summary, this article provides an example of using data mining to investigate a complex issue related to children's healthcare. Data mining results and clinical expertise were combined to develop a computer application to support practitioners in deciding the level of distraction intervention or care needed by a child undergoing a medical procedure and tailor instructions based on characteristics and preferences of the parent and child. Collaborative research teams consisting of nurse researchers, traditional statisticians, and scientists with data mining skills can help to transform information from large data sets into clinically meaningful knowledge to support decision making.

\section{REFERENCES}

1. McCarthy AM, Kleiber C. A conceptual model of factors influencing children's responses to a painful procedure when parent distraction is provided. Nurs Res. 2006;59:407-416.

2. von Baeyer CL, Marche TA, Rocha EM, Salmon K. Children's memory for pain: overview and implications for practice. J Pain. 2004;5:241-249.

3. Zempsky WT, Schechter NL. What's new in the management of pain in children. Pediatr Rev. 2003;24:337-348.

4. Taddio A, Katz J. The effects of early pain experience in neonates on pain responses in infancy and childhood. Paediatr Drugs. 2005;7: 245-257.

5. Bruce J, Davis EP, Gunnar MR. Individual differences in children's cortisol response to the beginning of a new school year. Psychoneuroendocrinology. 2002;27:635-650.

6. Kudielka BM, Buske-Kirschbaum A, Hellhammer DH, Kirschbaum C. HPA axis responses to laboratory psychosocial stress in healthy elderly adults, younger adults, and children: impact of age and gender. Psychoneuroendocrinology. 2004;29:83-98.
7. Kleiber C, Harper DC. Effects of distraction on children's pain and distress during medical procedures: a meta-analysis. Nurs Res. 1999;48:44-49.

8. Uman LS, Chambers CT, McGrath PJ, Kisely SA. A systematic review of randomized controlled trials examining psychological interventions for needle-related procedural pain and distress in children and adolescents: an abbreviated Cochrane review. J Pediatr Psychol. 2008;33:842-854.

9. Abbott PA, Zytkowski ME. In: Englebardt SP, Nelson R, eds. Health Care Informatics: An Interdisciplinary Approach. St Louis, MO: Mosby; 2002:115-128.

10. McCarthy AM, Kleiber C, Hanrahan K, Zimmerman MB, Westhus N, Allen S. Factors explaining children's responses to intravenous needle insertions. Nurs Res. 2010a;59:407-416.

11. Jay SM, Elliott C. Observation Scale of Behavioral DistressRevised. Unpublished Scoring Manual. Los Angeles, CA: Psychosocial Program, Children's Hospital of Los Angeles; 1986.

12. Hanrahan KS, McCarthy AM, Kleiber C, Lutgendorf S, Tsalikian E. Strategies for salivary cortisol collection and analysis in research with children. Appl Nurs Res. 2006;19:95-101.

13. McCarthy AM, Hanrahan K, Kleiber C, Zimmerman MB, Lutgendorf S, Tsalikian E. Normative salivary cortisol values and responsivity in children. Appl Nurs Res. 2009;22:54-62.

14. Kazak AE, Penati B, Waibel MK, Blackall GF. The perception of procedures questionnaire: psychometric properties of a brief parent report measure of procedural distress. J Pediatr Psychol. 1996;21: 195-207.

15. Aradine CR, Beyer JE, Tompkins JM. Children's pain perception before and after analgesia: a study of instrument construct validity and related issues. J Pediatr Nurs. 1988;3:11-23.

16. Beyer JE, Aradine CR. Content validity of an instrument to measure young children's perceptions of the intensity of their pain. I Pediatr Nurs. 1986;1:386-395.

17. Kleiber C, McCarthy AM, Hanrahan KS, Myers L, Weathers N. Development of the Distraction Coaching Index. Child Health Care. 2007;36:219-235.

18. McCarthy AM, Kleiber C, Hanrahan K, Zimmerman MB, Westhus N, Allen S. Impact of parent-provided distraction on child responses to an IV insertion. Child Health Care. 2010b;39: 125-141.

19. Tan P, Steinbach M, Kumar V. Introduction to Data Mining. Boston, MA: Pearson Addison Wesley; 2005.

20. Witten IH, Frank E. Data Mining: Practical Machine Learning Tools and Techniques. 2nd ed. San Francisco, CA: Morgan Kauffman; 2005.

21. Hall MA. In: Langley P, ed. Proceedings of the 17th International Conference on Machine Learning. San Francisco, CA: Morgan Kaufmann Publishing, Inc; 2000:359-366.

22. Hall M, Frank E, Holmes G, Pfahringer B, Reutemann P, Witten IH. The WEKA Data Mining Software: An Update. ACMSIGKDD Explorations Newsletter. New York, NY: ACM; 2009;11.

23. Smola AJ, Schölkopf B, Müller KR. The connection between regularization operators and support vector kernels. Neural Netw. 1998;11:637-649.

24. Berger AM, Berger CR. Data mining as a tool for research and knowledge development in nursing. Comput Inform Nurs. 2004; 22:123-131. 